ISSN 0103-9954

\title{
ESTIMATIVAS DE ÁREA BASAL E USO DO RELASCÓPIO DE BITTERLICH EM AMOSTRAGEM DE FLORESTA ESTACIONAL DECIDUAL
}

\section{ESTIMATES OF BASAL AREA AND THE USE OF BITTERLICH'S RELASCOPE FOR SAMPLING SEASONAL DECIDUOUS FOREST}

\author{
André R. Terra Nascimento ${ }^{1}$ Aldicir Scariot ${ }^{2} \quad$ José Alves da Silva $^{2} \quad$ Anderson C. Sevilha $^{3}$
}

\section{RESUMO}

A Floresta Estacional Decidual da região nordeste de Goiás é um tipo de vegetação florestal do grande bioma Cerrado, que pode ocorrer em áreas de afloramento de calcário e, geralmente, apresenta maior biomassa de espécies arbóreas que a vegetação de cerrado adjacente. Este estudo foi desenvolvido em uma área de floresta intacta (Fazenda Flor Ermo) e em quatro áreas de floresta perturbada (Fazendas Formosa, Traçadal, Manguinha e Conçeição Mocambo). O objetivo foi estimar a área basal e a intensidade amostral utilizando o relascópio de Bitterlich em florestas deciduais. A área basal foi de $29,3 \mathrm{~m}^{2} / \mathrm{ha}$ na floresta intacta e de 23 a $24 \mathrm{~m}^{2} /$ ha nas perturbadas. As florestas com perturbação acentuada apresentaram curvas de distribuição do desvio padrão dos pontos de amostragem com pequena variação comparada com a curvas das áreas com baixo nível de perturbação. Para um limite de confiança de $95 \%$ de probabilidade de confiança para a estimativa da média, 20 unidades amostrais com o relascópio podem ser suficientes para amostrar satisfatoriamente a área basal nesse tipo de vegetação.

Palavras-chave: intensidade amostral; inventário florestal; amostragem por pontos; floresta estacional decidual.

\begin{abstract}
The Deciduous Seasonal Forest of the Northeast region of the state of Goiás is one of the few types of forest formations in the Cerrado biome, which may occur in areas of limestone outcrops. Generally it has a higher biomass of tree species than the contiguous cerrado. This study was carried out in an undisturbed area of forest (Flor Ermo Farm) and in others four areas of disturbed forests: Formosa, Traçadal, Manguinha and Conceição Mocambo Farm. The study aimed to estimate the basal area and the sampling intensity needed to use Bitterlich's relascope in the deciduous forests. Estimates of basal area of the plots ranged from 23 to 24 $\mathrm{m}^{2} / \mathrm{ha}$ in the disturbed forests up to a maximum of $29.3 \mathrm{~m}^{2} / \mathrm{ha}$ in the undisturbed one. Forests with high disturbance showed very small variation of the standard deviation curve for the sampled points when compared to the area with low disturbance level. For a confidence level of $95 \%$ estimate of the mean, 20 sampling points with the relascope can be enough to sample the basal area in this kind of vegetation.
\end{abstract}

Key words: sampling intensity; forest inventory; point sampling; seasonal deciduous forest.

\section{INTRODUÇÃO}

A intervenção antrópica nas florestas deciduais da região da Bacia do Rio Paranã $\left(11^{\circ} 30^{\prime}-15^{\circ} 30^{\prime} \mathrm{S}\right.$ e $46^{\circ}-48^{\circ} \mathrm{W}$ ), no nordeste de Goiás e sudeste de Tocantins, ocorreu de forma mais acentuada nas três últimas décadas, com a extração de espécies arbóreas madeiráveis e a implantação de pastagens para o gado. Nesta região ocorrem florestas estacionais deciduais e semideciduais, que estão entre as extensões de florestas tropicais mais seriamente devastadas de todo o mundo (FAO, 1993; Whitmore, 1997). De 1988 a 1993, mais de $21 \%$ da vegetação original foi removida (Luíz, 1998) e mesmo recentemente, o desmatamento não parou, sendo que a cobertura florestal foi reduzida de 15 para 5\%, entre 1991 e 1999, em uma área amostral de 181.000 ha no Vale do Rio Paranã (Andahur, 2001), resultando em uma paisagem antropizada com fragmentos de vegetação em distintos níveis de perturbação e sucessão secundária.

1. Engenheiro Florestal, MSc., Doutorando em Ecologia pelo Instituto de Biociências, Universidade de Brasília, CEP 70910-900, Brasília (DF). arterra@unb.br

2. Engenheiro Florestal, PhD., Técnico da Embrapa Recursos Genéticos e Biotecnologia, Av. W3 Norte, s/n., Caixa Postal 02372, CEP 70770-900, Brasília (DF).

3. Biólogo, MSc., Técnico da Embrapa Recursos Genéticos e Biotecnologia, Av. W3 Norte, s/n., Caixa Postal 02372 , CEP 70770-900, Brasília (DF).

Recebido para publicação em 20/12/2002 e aceito em 4/10/2004. 
A fragmentação dos ecossistemas tropicais tem resultado em extensas áreas antropizadas, deixando florestas em diferentes níveis de perturbação e estágios de sucessão vegetal. As formações secundárias, naturais ou originadas pela intervenção antrópica (sensu Brown e Lugo, 1990; Finegan, 1997), são importantes na manutenção da diversidade biológica em escala regional e no aumento da conectividade entre fragmentos isolados na matriz da vegetação.

As florestas estacionais apresentam menor resilência que as florestas tropicais úmidas (Ewel, 1983) e um efeito de borda pouco evidente (Sampaio, 2001). A remoção de diversos indivíduos das classes superiores de diâmetro sem nenhuma preocupação com algum tipo de manejo em regime sustentável ou de práticas de conservação destas espécies é uma atividade constante nesse tipo de vegetação. Como essa vegetação é pouco estudada e está sendo rapidamente desmatada, são necessários métodos de amostragem rápidos, eficientes e de baixo custo que possam ser aplicados para inventários florestais, envolvendo estimativas de área basal e de volume, o que pode ser atingido com o uso da relascopia. Nesse sentido, Schneider et al. (1982) utilizaram o relascópio de banda larga para a cubagem de árvores individuais no inventário de florestas tropicais em Roraima, alcançando resultados altamente eficientes para aquelas condições de trabalho. A teoria e prática da relascopia têm mostrado que estão na dependência direta do diâmetro médio do povoamento, de sua homogeneidade e do erro máximo admissível para a amostragem. Como variam os diâmetros em cada povoamento e, conseqüentemente, sua homogeneidade, compreende-se que o número de parcelas por unidade de área também deve variar. Assim, quanto mais heterogênea e irregular a floresta, maior deve ser o número de pontos amostrais para estimar a área basal. De acordo com Bitterlich (1960), o número de pontos de amostragem reduz-se em cerca de 30\%, se em lugar do fator de área basal $\mathrm{K}=4$ usar $\mathrm{K}=2$.

Moscovich et al. (1999), comparando cinco diferentes métodos de amostragem em uma Floresta Ombrófila Mista, demonstraram que os métodos de Bitterlich e Prodan apresentaram estimativas acuradas dos parâmetros analisados (área basal, volume comercial e número de árvores por hectare), com boa eficiência relativa na estimativa dos parâmetros considerados. Embora os autores não tenham encontrado diferenças estatísticas significativas a $1 \%$ de probabilidade para os métodos em relação ao valor paramétrico, o método de Biterlich destacou-se em segundo lugar nas estimativas de área basal e do volume por hectare da floresta.

O presente estudo objetiva: 1. Estimar a área basal da comunidade arbórea em cinco áreas de floresta estacional decidual com o uso do Relascópio de Espelho de Biterllich. 2. Comparar as estimativas de área basal em florestas intacta e explorada. 3. Analisar as estimativas dos parâmetros estatísticos, indicando o esforço necessário para amostrar essa vegetação.

\section{MATERIAL E MÉTODOS}

\section{Descrição das fitofisionomias}

As florestas estacionais são formações que apresentam fisionomia variada e uma composição de espécies florísticamente relacionadas a estas comunidades, formando diferentes faixas de intergradação na vegetação. A porcentagem de deciduidade é variável em todas as formações, e o solo da floresta não apresenta um sub-bosque contínuo, sendo comum espécies vegetais arbustivas (Breedlove, 1973). A Floresta Estacional Decidual é um dos tipos de vegetação do grande bioma Cerrado e atinge elevados valores de cobertura dos elementos arbóreos (Ribeiro e Walter, 1998). Dentre as formações estacionais, as florestas deciduais representam um ecossistema sob grande pressão para a extração seletiva de espécies madeiráveis e o estabelecimento de novas áreas de pastagem para o gado (Scariot e Sevilha, 2000).

Na região nordeste de Goiás, município de São Domingos, a vegetação do Vale do Rio Paranã é composta principalmente de Cerrado (sensu stricto), Florestas Estacionais Deciduais e Semideciduais. As florestas estacionais deciduais predominavam nas áreas de afloramento de calcário e vales de solos distróficos. A ocupação dos vales ocorreu de forma desordenada, com a retirada de madeiras de qualidade como Myracrodruon urundeuva Fr. Allem. (aroeira), Schinopsis brasiliensis Engl. (braúna), Tabebuia impetiginosa Mart. ex. DC. (ipê-roxo), Cedrela fissilis Vell. (cedro), Hymenaea courbaril L. (jatobá), 
Aspidosperma subincanum Mart. (pereiro) e Amburana cearensis (Fr. Allem.) A. C. Smith. (cerejeira) (Scariot e Sevilha, 2000).

Os solos são férteis, evoluídos de calcário. O clima da região é do tipo $\mathrm{CW}$, segundo a classificação de Köppen, com as médias anuais de temperatura de $23^{\circ} \mathrm{C}$, pluviosidade média de $1400 \mathrm{~mm}$ e umidade relativa do ar em torno de $60 \%$. A temperatura do mês mais quente (outubro) é de $25^{\circ} \mathrm{C}$ e do mês mais frio, de $18^{\circ} \mathrm{C}$. A pluviometria do mês mais chuvoso (janeiro) situa-se em $225 \mathrm{~mm}$ com um período de três meses com deficiência hídrica (IBGE, 1994).

\section{Áreas amostradas}

Este estudo foi desenvolvido em cinco fragmentos (Tabela 1) de floresta estacional decidual: um intacto (Fazenda Flor Ermo) e quatro perturbados pela extração de madeira (Fazendas São Domingos, Conceição do Mocambo, Formosa e Manguinha), no município de São Domingos, região nordeste do estado de Goiás.

TABELA 1: Locais de amostragem, coordenadas geográficas e grau de intervenção de cada floresta estacional decidual amostrada, São Domingos, Goiás.

TABLE 1: Sampling areas, geographical coordinates and level of intervention in each sampled area of the seasonal deciduous forest, São Domingos, Goiás State.

\begin{tabular}{l|cccc}
\hline Fragmentos & Coordenadas geográficas & Nível Perturbação & Tempo Perturbação \\
\hline Flor Ermo & $13^{\circ} 20^{\prime} ; \mathrm{S} 46^{\circ} 35^{\prime} \mathrm{W}$ & sem & \\
São Domingos & $13^{\circ} 39^{\prime} 22^{\prime}, \mathrm{S} ; 46^{\circ} 45^{\prime} 28^{\prime}, \mathrm{W}$ & intermediária & 18 anos \\
Conceição do Mocambo & $13^{\circ} 37^{\prime} 40^{\prime}, \mathrm{S} ; 46^{\circ} 37^{\prime} 52^{\prime}, \mathrm{W}$ & intermediária & 15 anos e 1 ano \\
Formosa & $13^{\circ} 37^{\prime} 14^{\prime}, \mathrm{S} ; 46^{\circ} 47^{\prime} 31^{\prime}, \mathrm{W}$ & Pesada & 5 anos \\
Manguinha & $13^{\circ} 38^{\prime} 23^{\prime}, \mathrm{S} ; 46^{\circ} 47^{\prime} 06^{\prime}, \mathrm{W}$ & pesada & $3-5$ anos \\
\hline
\end{tabular}

Os níveis de cobertura entre as florestas são variáveis e refletem, principalmente, a época da perturbação e a retirada seletiva, ou não, de madeira da floresta. Na fazenda São Domingos, de exploração mais antiga, foi retirada apenas $M$. urundeuva, enquanto que, na Conceição do Mocambo, foram retiradas também Machaerium scleroxylum Tul. (pau-ferro) na primeira exploração, que também foi a principal espécie explorada na segunda exploração. Nas Fazendas Formosa e Manguinha foram exploradas todas as espécies madeiráveis, sendo que, na Manguinha, o fogo é um fator recorrente na floresta.

\section{Estimativas de área basal e número de árvores das comunidades com o relascópio}

Em cada área estudada (Tabela 1), foram feitas estimativas do número de árvores e da área basal por hectare. Para esta análise, foram mensuradas as árvores em 20 pontos amostrais sistemáticos com o Relascópio de Espelho de Bitterlich. Foram efetuadas estimativas com as bandas 1, 2 e 4, sendo selecionada a banda 2 por incluir um número mediano de indivíduos na amostragem com menores valores de desvio padrão entre as parcelas. Em cada ponto de amostragem, foram incluídos (pelo critério do DAP) todos os indivíduos aparentemente maiores que a Banda 2 e estimada a área basal por hectare.

$\mathrm{O}$ método da Relascopia consiste em contar as árvores, em um giro de $360^{\circ}$, cujos diâmetros à altura do peito (DAP) são iguais ou superiores a $(2 \operatorname{sen} \theta / 2)$, sendo $(\theta)$ um ângulo fixo, cujo vértice é o ponto central da unidade amostral. A seleção das árvores é, portanto, efetuada com probabilidade proporcional à área basal, ou ao quadrado do diâmetro e à freqüência (Pellico Neto e Brena, 1997). As árvores-limite, cujo DAP é igual ao ângulo de visada, devem ter suas distâncias horizontais controladas e medidas com a trena.

Em cada uma das parcelas de amostragem, foram contados os tocos existentes (testemunhas da retirada de madeira) para se ter uma idéias da extração e como um critério para auxiliar a diferenciação entre as áreas perturbadas e as intactas (Tabela 2). O maior número de tocos contados indicou as áreas com intervenção mais acentuada.

Considerando-se que as áreas são fragmentos de vegetação isolados e pertencem à mesma físionomia florestal, optou-se pela amostragem sistemática, sobre linhas perpendiculares, buscando cobrir a maior área possível em cada fragmento. 
Foi analisado o comportamento do desvio padrão (s), coeficiente de variação (C.V.) e o erro padrão da média de cada área, conforme aumentava o número de parcelas, sendo interpretado como um critério de intensidade de amostragem para este tipo de vegetação.

\section{Análise estatística da amostragem}

A amostragem foi efetuada na estação seca (agosto e setembro), durante a qual grande parte das espécies encontravam-se decíduas e o sub-bosque mais aberto e com uma maior radiação atingindo o piso da floresta, cujo dossel está bastante aberto nesta estação do ano.

Para se definir o tamanho da amostra (Snedecor e Cochran, 1980) e estimar a média $\mu$ (parâmetro) de uma população, estabeleceu-se:

Um limite superior (L) para que o erro pudesse ser tolerado na estimativa do parâmetro $\mu$ pela média amostral, ou seja $|\bar{x}-\mu| \cong$ L. Neste caso, o erro máximo refere-se à amostragem dos vinte pontos em cada fragmento, o qual variou de 0,5 até $3,0 \mathrm{~m}^{2}$ por hectare, considerando-se as estimativas de $1-\alpha=0,95$ e 0,99 .

A probabilidade desejada para que o parâmetro estivesse dentro dos limites de erro.

Um valor assumido para o desvio padrão da população $(\sigma)$.

A partir das estimativas de área basal para cada floresta, utilizaram-se os programas SAS (1990) e STATISTICA (1995) para simulações do erro padrão e do desvio das estimativas de área basal nas cinco áreas amostradas com os níveis de probabilidade de $1-\alpha=0,95$ e $1-\alpha=0,99$, sendo estas estimativas interpretadas como um critério importante da intensidade amostral, uma vez que inexistem dados para o tipo de formação florestal estudado.

Com base nos 20 pontos amostrais por fragmento e com os valores dos parâmetros estatísticos da amostragem (média, desvio padrão, erro padrão amostral percental) e considerando a formulação matemática anteriormente citada, simulou-se o comportamento da intensidade amostral com o aumento do número de parcelas para os dois níveis de probabilidade.

A homogeneidade das variâncias foi testada utilizando-se o teste de Bartlett's, e, posteriormente, as médias de área basal foram comparadas pelo teste de Tukey com 0,5 de probabilidade (Zar, 1999).

\section{RESULTADOS E DISCUSSÃO}

Não houve diferenças significativas entre as médias da área basal entre as cinco áreas estudadas (Tabela 2), embora ocorressem variações de 5,3 e 6,3 $\mathrm{m}^{2} /$ hectare entre Flor Ermo e as áreas mais intensamente exploradas para retirada de madeira (Formosa e Manguinha). O número de pontos $(\mathrm{n}=20)$ utilizados foi suficiente para obter um erro amostral percentual menor que $5 \%$ da média estimada (Tabela 2), com $95 \%$ de probabilidade de confiança.

TABELA 2: Area basal (G), erro de amostragem percentual (entre parênteses) e coeficiente de variação $(\mathrm{CV})$, média e total de tocos $(\mathrm{n}=20$ pontos por área), São Domingos, Goiás.

TABLE 2: Basal area $(\mathrm{G})$, relative sampling error (between brackets) and variation coefficient $(\mathrm{CV})$, mean and total of stumps $(\mathrm{N}=20$ points per area), São Domingos, Goiás State.

\begin{tabular}{l|c|c|c|c}
\hline Fragmentos & $\mathrm{G}\left(\mathrm{m}^{2} /\right.$ hectare $)$ & $\mathrm{C} . \mathrm{V}$. & Média de Tocos & Total de Tocos \\
\hline Flor Ermo & $29,3 a( \pm 4,4 \%)$ & 16,05 & 0,10 & 2 \\
Conceição do Mocambo & $28,8 a( \pm 2,2 \%)$ & 10,83 & 2,25 & 45 \\
São Domingos & $24,6 a( \pm 3,3 \%)$ & 15,17 & 0,15 & 3 \\
Formosa & $24,1 a( \pm 3,0 \%)$ & 15,14 & 3,00 & 60 \\
Manguinha & $23,0 a( \pm 4,4 \%)$ & 19,30 & 1,75 & 35 \\
\hline
\end{tabular}

Em que: $a=$ Médias seguidas pela mesma letra na vertical, não diferem significativamente pelo Teste de Tukey (P $\leq$ $0,05)$.

O coeficiente de variação envolvendo as cinco áreas estudadas variou de 10,83 (Conçeição do Mocambo, que é a área de exploração madeireira mais recente) a 19,3 (Manguinha, onde ocorreu a exploração madeireira mais intensa). Em Manguinha, ocorrem grandes clareiras, resultantes da retirada de 
árvores, formando um mosaico vegetacional com trechos de floresta mais desenvolvidos e outros bastante abertos.

O número de tocos (testemunho das árvores removidas), ainda vísiveis, variou bastante entre as áreas. Isto, provavelmente, seja resultado do tempo decorrido da exploração, visto que espécies mais resistentes à decomposição (ex: Myracrodruon urundeuva e Astronium fraxinifolium) possivelmente foram amostradas em todas as áreas. Estes valores são também afetados pela intensidade de remoção e pela densidade de determinadas espécies exploradas (espécies de rápida versus demorada decomposição).

As curvas com os erros padrão da média e desvio padrão revelaram inclinações semelhantes, conforme aumentava a intensidade amostral (Figuras 1 e 2), com exceção de Flor Ermo (E) e São Domingos (D). Estas duas áreas, com respectivamente 29,3 e $24,6 \mathrm{~m}^{2} /$ hectare de área basal, embora tivessem valores similares de coeficiente de variação (Tabela 2), apresentaram curvas com maiores variações iniciais no comportamento. Estas áreas, devido a maior amplitude diamétrica, necessitaram de maior esforço amostral que as outras para que a variação entre amostras pudesse se estabilizar (Figura 1).

As distribuições em diâmetro para espécies madeiráveis (em áreas intacta e explorada) da floresta estacional decidual, neste mesmo local, apresentaram curvas com um comportamento diferenciado. Espécies como Myracrodruon urundeuva e Astronium fraxinifolium, que foram altamente exploradas na região, tiveram uma redução considerável na densidade de indivíduos adultos na floresta explorada em comparação com a área intacta (Bueno et al., 2001).

As curvas que representam o erro padrão da média estimada (Figura 2), denotam características similares para os níveis de probabilidade estabelecidos. O número de pontos necessários, ao nível de probabilidade de $1-\alpha=0,95$, para se obter um erro padrão de $\pm 1,0 \mathrm{~m}^{2} /$ há, variou de 40 a 80 unidades nas áreas exploradas até 90 unidades na área intacta. A amostragem de 20 pontos foi suficiente para se obterem baixos erros padrões de 1,4-2,0 e 1,8-2,6 m²/ha nas áreas exploradas e de 2,0 e 2,8 $\mathrm{m}^{2} / \mathrm{ha}$ na área intacta (Flor Ermo), para níveis de probabilidade de $1-\alpha=0,95$ e $1-\alpha=0,99$. Em florestas desenvolvidas, como a Flor Ermo e mesmo São Domingos (onde a exploração ocorreu há 18 anos e a remoção de madeiras foi inferior às outras áreas exploradas), existe uma grande amplitude diamétrica, sendo, necessário, portanto, realizar uma maior intensidade amostral.

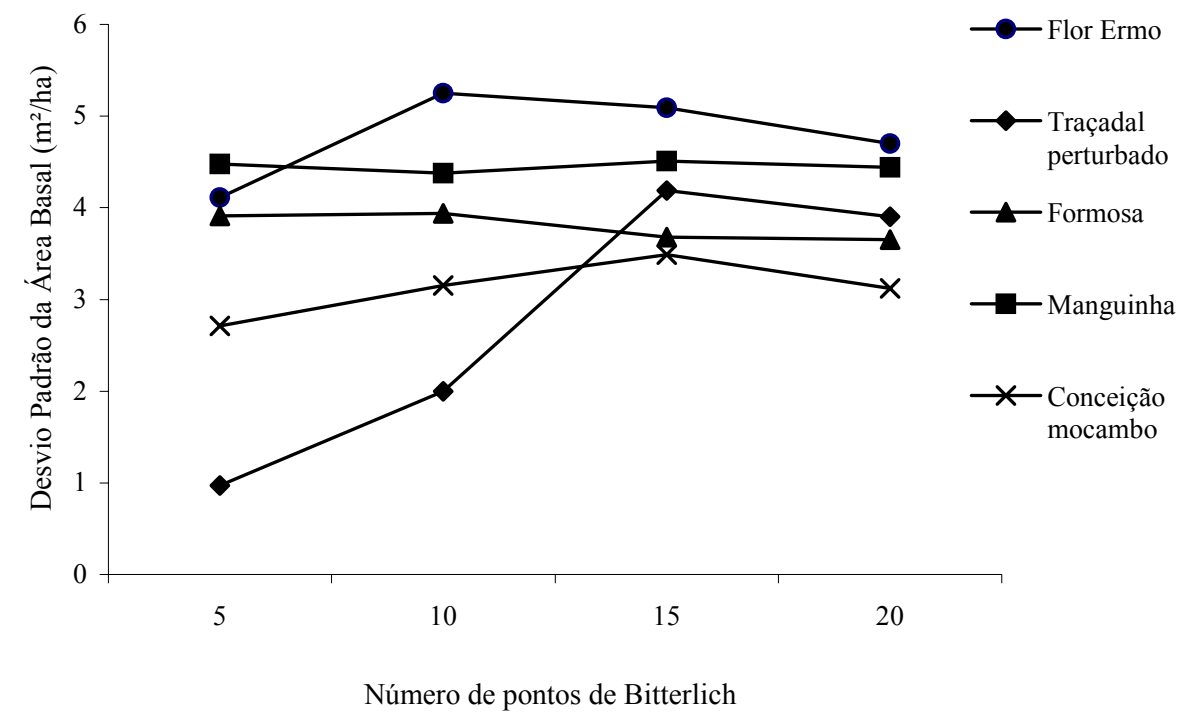

FIGURA 1: Desvio padrão da área basal média, estimado com o Relascópio de Bitterlich em relação à intensidade da amostragem, em florestas estacionais deciduais (Nordeste de Goiás).

FIGURE 1: Standard error of the mean basal area estimated with the Bitterlich Relascope related to the sampling intensity, in seasonal deciduous forest (Northeast Goiás). 
A área basal por hectare de um povoamento é a variável mais importante para a caracterização da densidade de um estoque de crescimento. Em povoamentos naturais maduros, o incremento em área basal e o incremento em volume mostram um desenvolvimento altamente paralelo (Loetsch et al., 1973). Desta forma, as árvores da floresta podem distribuir uma maior área basal em um menor número de indivíduos que pertençam às classes de maiores dimensões da distribuição em diâmetro.

Considerando o nível de probabilidade de 0,95 para a estimativa da média (Figura 2), as 20 unidades amostrais podem ser indicadas como uma estimativa razoável, com um erro padrão da média da área basal menor que $2,0 \mathrm{~m}^{2} /$ ha, para este tipo de vegetação. Para estimativas mais acuradas (1- $\alpha=0,99$ e um erro padrão mínimo de $1 \mathrm{~m}^{2}$ por hectare), incomum em amostragens de florestas heterogêneas, deve-se aumentar a intensidade da amostragem.
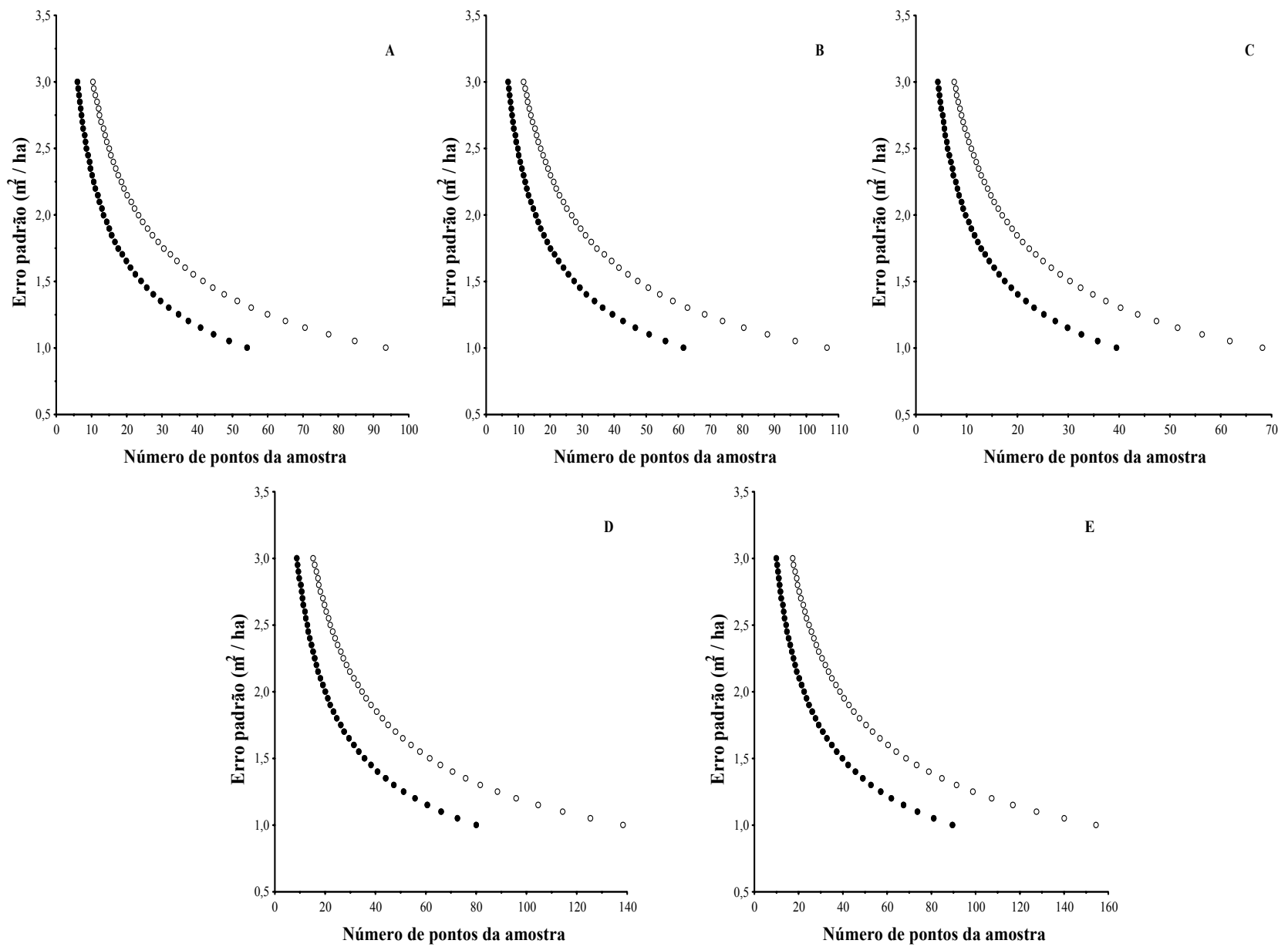

FIGURA 2: Erro padrão das estimativas da média com o incremento do número de pontos amostrados, Floresta Estacional Decidual, nordeste de Goiás. Círculos cheios representam níveis de probabilidade de $1-\alpha=0,95$ e círculos vazios, de $1-\alpha=0,99$. Notar os valores do eixo $\mathrm{x}$, que representam a intensidade da amostragem para cada área nos dois níveis de probabilidade estabelecidos. Áreas amostradas - Florestas exploradas: Formosa (A), Manguinha (B), Conceição do Mocambo (C), Fazenda São Domingos (D); e floresta intacta: Flor Ermo (E).

FIGURE 2: Standard error of the mean estimated in relation to the increasing number of sampling points, Seasonanl Deciduous Forest, Northeast Goias. Filled circles represent probability level of 1- $\alpha=$ 0,95 and empty circles level of $1-\alpha=0,99$. Note that values under $\mathrm{x}$-axis represent sampling intensity for each site with both levels of probability. Sampled sites: Disturbed forests: Formosa (A), Manguinha (B), Conceição do Mocambo (C), Fazenda São Domingos (D); and intact forest: Flor Ermo (E). 


\section{CONCLUSÕES}

Com base nos resultados obtidos e para as condições da Floresta Estacional Decidual do nordeste de Goiás, pode-se concluir:

O uso de relascópio de espelho de Bitterlich foi um instrumento adequado para estimar a área basal com praticidade e precisão, com baixos valores para o erro padrão da média. As estimativas de área basal variaram de $23,0 \mathrm{~m}^{2} /$ ha na floresta perturbada ate $29,5 \mathrm{~m}^{2} /$ ha na floresta intacta.

Não foram observadas diferenças estatísticas significativas entre as estimativas de área basal por hectare para as cinco áreas estudadas, embora ocorressem variações de até $6,3 \mathrm{~m}^{2}$ e 5,3 $\mathrm{m}^{2}$ por hectare entre a floresta intacta e as florestas perturbadas.

Foram encontrados baixos valores para o erro amostral percentual $(2,2$ a $4,4 \%)$ e para o coeficiente de variação $(10,8$ a $19,3 \%)$ da área basal nas cinco áreas, estando estes valores dentro dos limites normalmente estabelecidos para inventários em florestas naturais heterogêneas.

\section{AGRADECIMENTOS}

Este trabalho foi financiado pelo projeto "Conservação e Manejo da Biodiversidade em Fragmentos de Florestas Semidecíduas", financiado pelo PRONABIO/PROBIO, com o apoio do BIRD/GEF/CNPq e do Ministério do Meio Ambiente. André R. Terra Nascimento recebeu bolsa DTI do CNPq (processo $\mathrm{n}^{\circ}$ 380612/00-3). À Embrapa Recursos Genéticos e Biotecnologia, pelo apoio logístico. A Célia M. Cordeiro, pelo grande auxílio nas análises estatísticas. Ao professor Paulo Renato Schneider (UFSM), pelas importantes contribuições ao trabalho. A Daniel M. Vieira, Maurício B. Sampaio e Nilton F. Barbosa, pela ajuda na amostragem de campo.

\section{REFERÊNCIAS BIBLIOGRÁFICAS}

ANDAHUR, J.P.V. Floresta e questões de gestão ambiental na bacia do vale do Paranã. Dissertação de Mestrado. 2001. 92p. Dissertação (Mestrado em Ciências Florestais). Universidade de Brasília, Brasília.

BREEDLOVE, D. E. Phytogeography and vegetation of Chiapas (México). In: GRAHAM, A. (ed.) Vegetation and vegetational history of Northern Latin America. Amsterdan: Elsevier Scientific Publishers. 1973, p. 140-165.

BROWN, S. \& LUGO, A. E. Tropical secondary forests. Journal of Tropical Ecology, v. 6, p. 1-32, 1990.

BITTERLICH, W. Vorteile der variablen probekreistechnik. Allgemeine Forstzeitung. 8 p. 1960.

BUENO, P.C., SCARIOT, A.; SEVILHA, A.C. Estrutura populacional de espécies madeiráveis em áreas intacta e explorada de Floresta Decidual. Bol. Ezequias Paulo Heringer, 2001 (no prelo).

EWEL, J. Sucession. In: GOLLEY, F.B. (ed.) Tropical rain forest Ecosystems: structure and fuction. Amsterdam: Elsevier Scientific Publishing Company. 1983, p. 217-223.

FAO. Forest Resource Assessment 1990. Tropical countries. FAO Forestry Paper 112. FAO, Rome. 1993.

FINEGAN, B. Bases ecológicas para el manejo de bosques secundarios de las zonas húmedas del trópico americano, recuperación de la biodiversidad y producción sostenible de madera. In: MEMORIAS DEL TALLER INTERNACIONAL SOBRE EL ESTADO ACTUAL Y POTENCIAL DE MANEJO Y DESARROLO DEL BOSQUE SECUNDARIO TROPICAL EN AMÉRICA LATINA. Pucalpa, Peru, Anais..., 1997, p. 106-119.

IBGE Zoneamento Geoambiental e Agroecológico do Estado de Goiás: região nordeste/Hilton Lenzi Moreira (Coord.). Rio de Janeiro: Instituto Brasileiro de Geografia e Estatística, Divisão de Geociências do Centro-Oeste. 178 p- (Estudos e pesquisas em Geociências). 1994.

LOETSCH, F.; ZÖHRER, F.; HALLER, K.E. Forest inventory. 2 ed., Munique: BLV Verlagsgesellschaft. 1973, 469p.

LUÍZ, G. C. Estudo do impacto ambiental a partir da análise espaço-temporal - Caso da região Vão do Paranã GO. 1998. Dissertação (Mestrado em Geociências)- Universidade Federal de Goiás, Goiânia.

MOSCOVICH, F.A.; BRENA, D.A.; LONGHI, S.J. Comparação de diferentes métodos de amostragem de área fixa e variável, em um floresta de Araucaria angustifolia. Ciência Florestal, v.9, n.1, p.173-191, 1999.

PÉLLICO NETTO, S. \& BRENA, D.A. Inventário florestal. Curitiba: Editado pelos autores. 1997, 316 p. 
RIBEIRO, J. F. \& WALTER, B. M. T. Fitofisionomias do bioma Cerrado. In: SANO, S.M; ALMEIDA, S.P. (eds.) Cerrado: ambiente e flora. Planaltina: EMBRAPA-CPAC, 1998, p. 89-166.

SAMPAIO, A.B. Efeito de borda nas espécies arbóreas de uma floresta Estacional Decidual no vale do Paranã. 2001. 82 p. Dissertação (Mestrado em Ecologia). Universidade de Brasília, Brasília.

SAS Institute Inc., SAS Languace : Reference, Version 6, First Edition. Cary, NC : SAS Istitute Inc., 1990.1042 p.

SCARIOT, A. \& SEVILHA, A.C. Diversidade, estrutura e manejo de florestas deciduais e as estratégias de conservação. In: CAVALCANTI, T.B. \& WALTER, B.M.T. (Org.) Tópicos atuais em Botânica. Brasília: Sociedade Botânica do Brasil/Embrapa Recursos Genéticos e Biotecnologia. 2000, p.183-188.

SCHNEIDER P.R.; SILVA, J.A.; ANDRAE, F.; COZER, E.L. Estrutura e produção das florestas naturais ao longo do Rio Branco no território federal de Roraima. Ciência e Natura, v. 4, p. 115-127, 1982.

SNEDECOR, G.W. \& COCHRAN, W. C. Statistical methods. The Iowa State University Press: United States of America. 1980, $507 \mathrm{p}$.

STATSOFT, INC. STATISTICA for Windows. [Computer program manual]. Tulsa OK, 1995.

WHITMORE, T. C. Tropical forest disturbance, disappearance, and species loss. In: Laurance, W. F. \& R. O. Bierregaard, Jr. (eds.) Tropical Forest Remnants: Ecology, Management, and Conservation of Fragmented Communities. The University of Chicago Press: Chicago. 1997, p. 3-14.

ZAR, J. H. Biostatistical analysis. Prentice Hall: New Jersey. 1999, 663 p. 